\title{
Responsive Web Design Based on Different-Sized Devices
}

\author{
FENG Xing Li ${ }^{1, a}$, SUO Zhi Hai*1,b and LUO Jun Feng ${ }^{1, \mathrm{c}}$ \\ ${ }^{1}$ Data and Information Center, Xi'an Jiaotong University, Xi'an, Shaanxi, China \\ afengxingli5927@sina.com, *bzhsuo@mail.xjtu.edu.cn, 'luojf@mail.xjtu.edu.cn
}

Keywords: Responsive Web Design, Mobile Device.

Abstract. In order to adapt the Web page of the PC version to different-sized mobile devices automatically, reduce costs of web development and maintenance, and enhance the browsing experience of users, a responsive web with $\mathrm{Html} 5$ and Css3 language was designed and implemented in this paper . Through the simulation by Windows Resizer in Chrome, the approach achieves good results.

\section{Introduction}

With the popularity of 3G, more and more people are using mobile devices to surf the internet. As the terminal to access the internet, mobile devices become more and more common than desktop devices. Thus, the web designers have to face a problem: how to adapt the same web page to different-sized devices and different browsers.

Size of phone's screen is relatively small, its width is usually 600 pixels or less; The width of the screen of the PC is generally more than 1000 pixels (At present, the popular width is $1366 \mathrm{x} 768$ pixels), and some reached 2000 pixels. It is not an easy task to show the same content on different-sized screen with satisfactory layout.

The solution of most websites is to provide different web versions for different devices. Such as an iPhone version for iPhone that with 640 x 960 pixels or an iPad version for iPad that with 1024 x 768 pixels. On this way it's more trouble to maintain several versions of one web. And if the website has several portal, the complexity of the architecture design will be greatly increased.

Thus, it has long been considered that whether the same web can be automatically adapted to different-sized screen, and adjust the layout automatically according to the screen width.

In 2010, Ethan Marcotte proposed "Responsive Web Design" which refers to make appropriate adjustments by identifing the width of the screen automatically. ${ }^{[1]}$

\section{Media Queries}

The core of " responsive web design" is Media Query module which introduced from CSS3. ${ }^{[2]}$

That is, detecting screen width automatically and then the corresponding CSS file is loading.

$<$ link rel="stylesheet" type="text/css" media="screen and (max-device-width: 1024px)"

href="largeScreen.css" />

The code above means if screen width is shorter than 1024 px, then largeScreen.css is loading.

In addition to loading CSS file with the html tags, you can also load a css file in an existing CSS file. For example:

@import url("largeScreen.css") screen and (max-device-width: 1024px);

In the same CSS file, you can choose different CSS rules according to screen resolutions.

@media screen and (max-device-width: 480px) \{

.column \{

float: left;

width:auto;

\}

\#sidebar \{

display:none; 
The code above means if screen width is shorter than 480px, then column block is float left, and its width is adjusted automatically, and sidebar block is not show.

\section{Embracing Fluid Layouts}

Allow web page to automatically adjust the width. First, add a viewport label on the head of the web page.

$<$ meta name="viewport" content="width=device-width, initial-scale=1" / >

The code above represents that the width of web page equals to device width, and the original scale is $1.0 .{ }^{[3]}$

All of the popular broswer include IE9 support the configuration. For those browsers with low versions(mainly IE6/IE7/IE8), css3-mediaqueries.js is needed. The code is as follows ${ }^{[4]}$ :

$<$ !--[if lt IE 8] $>$

$<$ script

src="http://css3-mediaqueries-js.googlecode.com/svn/trunk/css3-mediaqueries.js" $></$ script $>$

$<$ ![endif]-->

No absolute width. The web page can adjust width according to device-width, so it's very important that the absolute width like width:xxx px cannot be used.

It can only be used in percentage like: width: $\mathrm{xx} \%$;

or width:auto;

Relative size of the font. The size of font cannot be absolutely, it must be relatively ${ }^{[5]}$. For example:

body \{

font: normal 100\% Helvetica, Sans-Serif;

The code above shows the font size is $100 \%$ of default font size.

h1 \{

font-size: 2.5em;

\}

The font size of h1 is 2.5 times of default size.

Fluid grid. Fluid grid is that every block is floating, not fixed. For example: float: right;

The benefit of element float is: if the width is too small to fit two elements, the back element will automatically scroll to the bottom of the front element, not overflow in the horizontal direction, which avoid the appearance of a horizontal scroll bar. ${ }^{[6]}$

In addition, use of absolute positioning (position: absolute) should be careful.

Fluid image. In addition to layout and text, " responsive Web Design" must also implement automatic scaling of picture. This only need one line code: img \{ max-width: $96 \%$; $\}$

And the code above also works for video embeded in web. So it can also be written into: img, object $\{$ max-width: 96\%;

But the IE browser of old version is not support max-width, so it can only be written into: img \{ width: 96\%; \}

In addition, When zooming the picture on windows, the image distortion may occur. In this case, you can try using IE's proprietary command:

img $\{$-ms-interpolation-mode: bicubic; $\}$

or using imgSizer.js written by Ethan Marcotte. ${ }^{[7]}$

addLoadEvent(function() \{

var imgs = document.getElementById("content").getElementsByTagName("img");

imgSizer.collate(imgs);

\}$)$; 


\section{Html5}

With the help of HTML5, it is possible to embed video and audio, high quality drawings, charts and animation and many other rich content without using any plugins and third party programmas as the functionality is built into the browser.

Now it is easy to see which parts of the page are headers, nav, etc as the tags like $<$ header $>,<$ nav $>$, etc are specific for these all and know what their meaning. By using HTML5 elements we can increase the semantic value of the web page as the codes are very standardized.

HTML 5 will enable web designers to use cleaner and neater code. we can remove most div tags and replace them with semantic HTML 5 elements. ${ }^{[8]}$

In summary, Coding with HTML5 will save much time.

\section{CSS3}

CSS3 is easier to make changes: you can modify individual modules, which are integrated with the overall system. The modular approach of CSS3 helps to develop and maintain the system based on the module.

Multi-Column Module is an important function of CSS3, which allows to place text in multiple columns. The advantage for users is that while reading the text in multiple columns you don't need to scroll up and down and side to side. Everything can be placed on the same page that layout makes it convenient and economical.

The concept of CSS allows user to attach information to CSS-style in a separate document or as an attachment within a HTML-document. You can also import multiple style sheets anywhere. Users can specify an alternative style sheets, choosing between them as needed. Above all, CSS3 enables responsive designs and more. ${ }^{[9]}$

\section{Example}

Responsive web designed in this paper is tested by Windows Resizer which can be downloaded from the following URL:

https://chrome.google.com/webstore/detail/kkelicaakdanhinjdeammmilcgefonfh, and installed in Chrome. ${ }^{[10]}$

The following example is tested in Chrome with 640 x 960 pixels wide viewport:

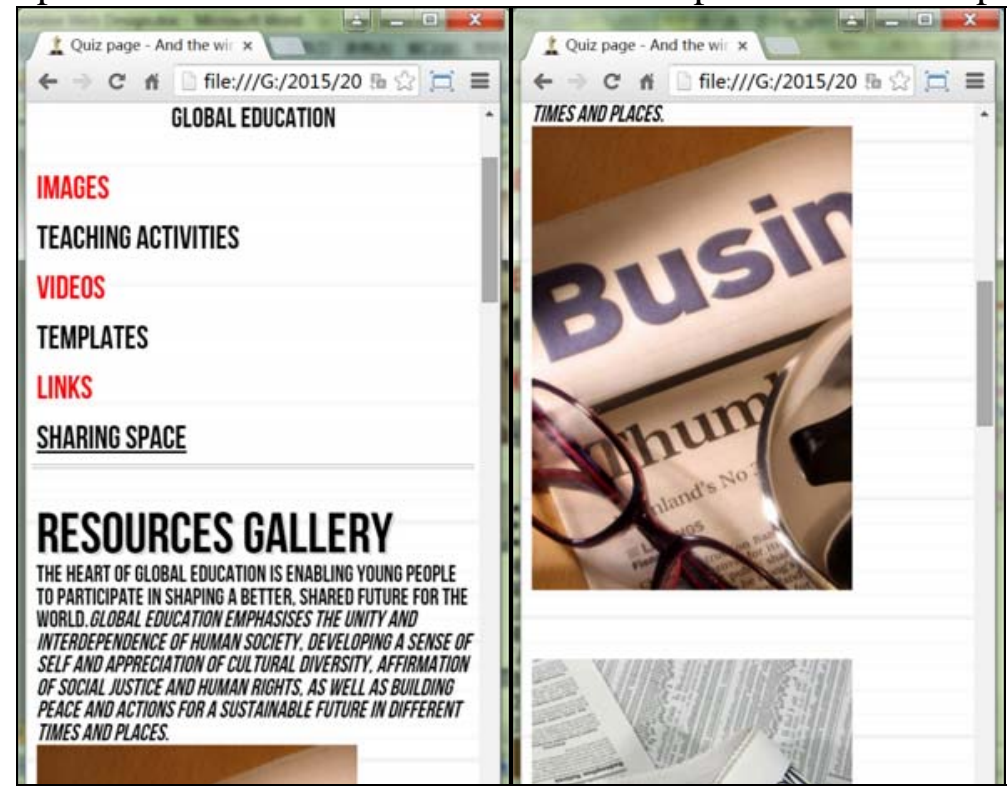

Fig. 1: Display of menu and images with 640 x 960 pixels

The follow example is tested in Chrome with 1024 x 768 pixels wide viewport: 


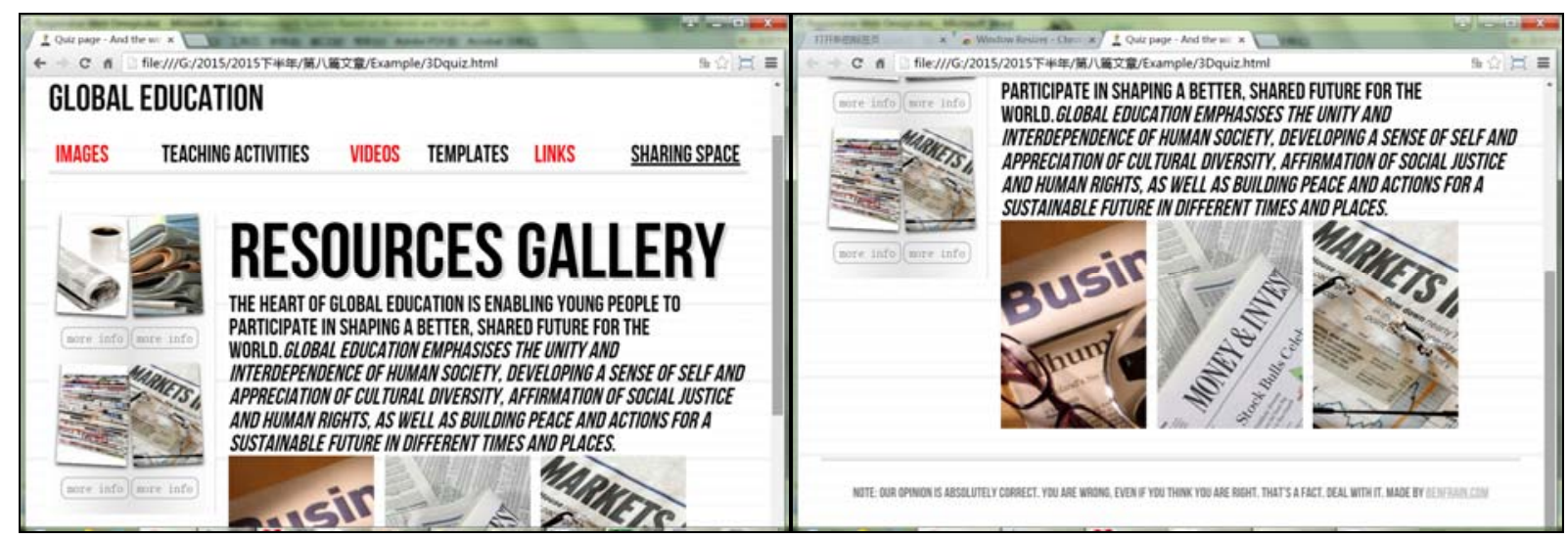

Fig. 2: Display of menu and images with 1024 x 768 pixels

In this example, When width is less than 768px, the top menu bar and the right three images will arrange vertically. When the width is greater than 1024 pixels, the top menu bar and the right three images will arrange horizontally. And the text section will also change with the screen width.

\section{Summary}

In order to provide a reasonable and beautiful visual experience and reduce the costs of re-development of website on small-screen mobile devices, the paper proposed the solution of responsive web design initially. And combined with the latest technology, a web that can be display on different-sized devices is designed and implemented Simulation testing using Windows Resizer in Chrome shows that web designed with the solution in this paper has very good user experience.

\section{Acknowledgements}

This work was financially supported by the Special Program of Ministry of Education, Science and Technology Development Center(No.2011jybkjfz01).

\section{References}

[1] Information on http://alistapart.com/article/responsive-web-design.

[2] Information on http://www.ruanyifeng.com/blog/2012/05/responsive_web_design.html.

[3] Y. Yang, L. Chen, Q.B. Dong: Mode. Educa. Tech. Vol. 23 (2013), p. 107.

[4] P. Gasston: The Modern Web: Multi-Device Web Development with HTML5, CSS3, and JavaScript (No Starch Press, USA, 2013).

[5] Information on http://isux.tencent.com/responsive-web-design.html.

[6] Information on http://alistapart.com/article/fluidgrids.

[7] Information on http://unstoppablerobotninja.com/demos/resize/imgSizer.js.

[8] Information on http://www.chinaz.com/web/2012/0724/264466.shtml.

[9] Information on http://www.educity.cn/jianzhan/630933.html.

[10] B. Frain: Responsive Web Design with HTML5 and CSS3 (Packt Publishing Ltd, UK, 2012). 